\title{
Strates
}

STRATES Matériaux pour la recherche en sciences sociales

15 | 2008

Union européenne - Voisinages. La quête d'une intégration régionale

\section{Les mouvements de personnes en Méditerranée : des migrations aux mobilités}

Philippe Fargues et Hervé Le Bras

\section{(2) OpenEdition}

Journals

Édition électronique

URL : http://journals.openedition.org/strates/6541

DOI : $10.4000 /$ strates.6541

ISSN : $1777-5442$

Éditeur

Laboratoire Ladyss

Édition imprimée

Date de publication : 1 janvier 2008

Pagination : 119-128

ISSN : 0768-8067

Référence électronique

Philippe Fargues et Hervé Le Bras, «Les mouvements de personnes en Méditerranée : des migrations aux mobilités », Strates [En ligne], 15 | 2008, mis en ligne le 22 janvier 2013, consulté le 08 septembre 2020. URL : http://journals.openedition.org/strates/6541 ; DOI : https://doi.org/10.4000/strates.6541

Ce document a été généré automatiquement le 8 septembre 2020

Tous droits réservés 


\title{
Les mouvements de personnes en Méditerranée : des migrations aux mobilités
}

\author{
Philippe Fargues et Hervé Le Bras
}

1 Avec une migration nette de plus de 1,8 million $^{1}$ contre 1,2 million pour l'Amérique du Nord, l'UE est désormais la première région d'immigration du monde. Tous les États membres se sont transformés les uns après les autres en pays d'immigration : sur les 25 États membres, il n'y en avait plus en 2005 parmi les Peco (Pays d'Europe centrale et orientale) que quatre qui conservaient, un an après leur adhésion, un solde migratoire négatif ${ }^{2}$. L'attraction de l'UE s'exerce d'abord sur sa périphérie, si bien que tous ses voisins - sauf la Norvège qui est évidemment un voisin très spécifique - ont un solde migratoire négatif avec elle. Cela est vrai pour les pays accédants (Bulgarie et Roumanie), les pays candidats (Croatie, Macédoine et Turquie), comme pour les simples pays tiers : la Russie (qui a un solde migratoire positif avec les anciens États de l'URSS, mais négatif avec le reste du monde dont l'UE) ${ }^{3}$, l'Ukraine, la Biélorussie et les pays de la rive sud de la Méditerranée ${ }^{4}$.

2 Ces développements sont en général mis en relation avec trois faits, la pression des pays pauvres du Sud en forte croissance démographique, la demande de travail des pays riches du Nord ou du Golfe persique, et la baisse tendancielle du coût de transport. Cet article montre que le premier élément doit être relativisé, que le second et le troisième, au contraire, dessinent les grandes lignes d'une nouvelle forme de migration qui changera sans doute les rapports entre les nations, en particulier dans l'espace euroméditerranéen. Il porte également l'accent sur des éléments nouveaux des mouvements de personnes en Méditerranée : le rôle de la qualification croissante des migrants, le fait que ces migrants sont de plus en plus considérés par les pays d'origine comme des ressources, le statut de pays d'immigration acquis depuis peu par ces pays de la rive sud, enfin la nécessité de prendre en compte les retours, très fréquents mais difficiles à comptabiliser, et surtout les allers-retours des populations migrantes. 
L'objectif du texte est de montrer que c'est en termes de mobilités et non plus de migrations classiques qu'il faut analyser ces mouvements.

\section{Réexaminer les facteurs des migrations méditerranéennes}

\section{La pression démographique de la rive sud : un facteur déclinant}

3 Avec une population jeune et une croissance démographique de 1,5\% par an, le dynamisme démographique des pays du Sud et de l'Est de la Méditerranée contraste avec la stabilité de la population européenne dont la croissance oscille maintenant autour de zéro. Ce différentiel va-t-il produire une "grande migration» pour reprendre le titre de l'essai à succès de Michaël Enzensberger?

4 Actuellement, le stock de migrants dans le monde, c'est-à-dire le nombre de personnes qui vivent dans un pays où elles ne sont pas nées, est de 160 millions de personnes, soit 2,5 \% seulement des 6,4 milliards d'humains. Dans ce total, l'Amérique du Nord compte pour 24 millions de migrants et l'Union européenne pour 18 millions. Viennent ensuite les pays du Golfe avec 7 millions de migrants puis un ensemble de pays où les motifs politiques l'emportent sur les raisons économiques : en Russie et dans les anciens État qui constituaient l'URSS, les populations sont restées mêlées avec des Russes dans le Caucase, en Ukraine et en Asie centrale, et inversement ces nationalités en Russie. En Iran, au Zaïre, au Pakistan, les migrants sont essentiellement des réfugiés. Les migrations actuelles, dont le volume total est de l'ordre de 5 millions de personnes par an, soit moins d'un humain sur mille, ne sont pas assez importantes pour faire évoluer rapidement la proportion mondiale de migrants. En tenant compte des retours qui comptent pour près du tiers, au rythme actuel, le stock de migrants dans le monde devrait se stabiliser autour de 300 millions de personnes, ce qui ne dépassera pas $5 \%$ de la population mondiale à long terme.

Le montant modeste des stocks et des flux de migrants dans le monde peut dissimuler des différentiels locaux importants, particulièrement autour de la Méditerranée en raison de la proximité de pays développés et en développement. C'est le thème bien connu des « pressions migratoires ». Il convient cependant de ne pas généraliser ce que montrent les reportages de la télévision aux Îles Canaries. Hania Zlotnik, directeur de la division de la population aux Nations unies, a clairement montré qu'il n'y a aucun rapport entre le taux de croissance démographique et le taux d'émigration ${ }^{5}$. Elle a comparé les valeurs des deux taux dans 120 pays du monde sur une longue période (1960-1995). La corrélation est même légèrement négative, ce qui va dans le sens inverse de l'idée de pression démographique : la population aurait tendance à se diriger vers les zones les plus denses - ou en tout cas celles qui connaissent une forte croissance démographique. C'est d'ailleurs ce que l'on observe à l'intérieur de nombreux pays et que l'on désigne par le terme de concentration urbaine. Les paysans chinois quittent les montagnes du Sud et les steppes de l'Ouest peu peuplées pour se regrouper sur les zones côtières déjà très denses. Les Américains délaissent le Middle West pour la Californie, la Floride ou la sun belt. La concentration de population a toujours été favorable au développement et l'a accompagné. On a rarement vu des zones désertiques attirer les foules et prendre la tête du progrès. Le seul cas où des migrations de grande ampleur pourraient apparaître est celui de migrations de 
détresse causées par de graves difficultés économiques ou politiques, mais elles aboutissent en général de l'autre côté de la frontière du pays concerné, comme on l'a vu dans le cas du Rwanda (réfugiés en Ouganda, Tanzanie et République du Congo) ou de l'Afghanistan (réfugiés dans la province de Meched en Iran et de Peshawar au Pakistan). De telles migrations ne sont pas prévisibles actuellement en Méditerranée.

6 Le fantasme de l'invasion est cultivé par l'extrême droite européenne. Mais les partis $\mathrm{du}$ centre et de la gauche entretiennent depuis peu le fantasme inverse que l'immigration pourrait enrayer le vieillissement au Nord de la Méditerranée. Mais, d'une part, les arrivées de jeunes actifs sur le marché du travail des pays du Sud de la Méditerranée, encore très nombreuses, vont rapidement se stabiliser (dès 2010 les pays arabes et la Turquie vont ressentir les effets sur l'emploi de la baisse de natalité amorcée dans les années 1980). D'autre part l'immigration de jeunes adultes ne saurait contrecarrer l'effet de l'allongement de la vie qui inéluctablement augmente la proportion de personnes de plus de 60 ans par rapport à celles d'âge actif (20 à 60 ans). Les Nations Unies ont calculé que si la France voulait conserver une proportion de trois personnes âgées de 15 à 60 ans pour une personne de plus de 60 ans, elle devrait accueillir chaque année 920000 immigrants - ce qui est peu envisageable compte tenu des très nombreux emplois qu'il faudrait alors créer.

\section{L'offre de travail au Nord restera un puissant facteur d'immigration}

7 Malgré le niveau élevé du chômage dans les pays de l'UE, une importante offre de travail suscite des flux migratoires en direction de l'UE. D'abord, le taux de croissance économique élevé de pays comme l'Espagne ou l'Irlande qui n'avaient pas connu d'immigration, a créé un appel d'air. Plus généralement, plusieurs facteurs se sont conjugués pour faire coexister chômage et immigration. La spécialisation plus poussée de la main-d'œuvre engendre des déficits locaux que ne comblent plus les migrations internes. Les migrations interrégionales à l'intérieur de l'Europe se sont stabilisées et décroissent même dans plusieurs pays pour deux raisons : l'augmentation forte des couples bi-actifs à cause de l'arrivée massive des femmes sur le marché du travail limite les changements de région de résidence car les deux membres du couple doivent simultanément changer d'emploi; en outre, les différences de coûts du logement et l'accroissement des formalités nécessaires pour changer de résidence agissent comme un frein supplémentaire à la migration européenne interne. Dans ces conditions, des secteurs d'activité manquent de main-d'œuvre dans telle région d'Europe alors que d'autres régions ont un chômage dans les mêmes spécialités - d'autant que les Européens au chômage sont dans l'ensemble peu mobiles. Le recours à une maind'œuvre étrangère plus disposée à la mobilité résout souvent la difficulté en alimentant un courant migratoire qui n'aurait pas de raison d'être si l'on raisonnait au niveau de l'ensemble de l'UE.

8 L'appel à l'immigration est aussi la conséquence des grands changements de structure de la main-d'œuvre dans l'Union européenne. D'une part, les emplois ouvriers ont diminué dans les pays les plus riches qui ont délocalisé leurs productions les plus lourdes et les moins spécialisées vers les Peco et maintenant vers la Roumanie et la Bulgarie. L'intégration à l'Union a eu l'effet inverse de l'intégration à l'Alena. L'ouverture des frontières en Europe n'a pas entraîné une mobilité des hommes mais des capitaux qui se sont investis dans les pays les plus pauvres de l'UE, favorisant leur 
développement et leur rattrapage. Au contraire, l'Alena a dopé l'immigration mexicaine vers les États-Unis en raison du différentiel de salaire très important. D'autre part, de nouveaux secteurs d'emploi sont apparus, particulièrement dans les services à la personne à cause du vieillissement de la population et dans le commerce et le secteur hôtelier à cause du tourisme. Enfin dans le bâtiment et les travaux publics, il est devenu de plus en plus difficile d'employer des nationaux qui rechignent devant le travail physique en plein air et les déplacements. Les trois secteurs de l'hôtellerie, du bâtiment et des services à la personne continuent dès lors à engager des demandeurs d'emploi venus du Sud et de l'Est de la Méditerranée. Il y a donc toutes les chances que l'émigration des pays arabes perdure, voire qu'elle s'accroisse dans les dix années à venir puisque le moteur d'appel (effet pull) continuera à se développer sauf récession sévère au Nord.

Comme l'Italie, puis l'Espagne, la Grèce et le Portugal naguère, les nouveaux pays membres vont vraisemblablement à leur tour faire appel à l'immigration au cours des dix prochaines années. En revanche, souvent évoquée, la diminution du nombre des actifs à l'horizon 2020 ne résiste pas à l'examen. Selon la projection moyenne des Nations Unies, la population âgée de 15 à 64 ans dans les 25 pays de l'UE passera de 310 millions de personnes en 2005 à 305 millions en 2020. Cette faible diminution de 5 millions de personnes ne devrait pas engendrer de flux migratoires car elle devrait être facilement absorbée par un léger redéploiement de la population active : il suffit par exemple que le nombre d'années travaillées augmente de 6 mois ou que le taux d'activité des femmes à 30 ans, qui est de $70 \%$, passe à $72,5 \%$ pour que la population active augmente de 5 millions de personnes. À horizon plus lointain, bien que l'on entre dans le domaine hypothétique, il est possible que la mondialisation de la migration franchisse un nouveau seuil avec l'arrivée de Chinois et d'Indiens. Le nombre de Chinois dans l'UE a déjà été multiplié par trois au cours des dernières années et ils s'installent en nombre en Algérie ${ }^{6}$. En règle générale, ce ne sont pas les plus pauvres qui émigrent ${ }^{7}$, mais les enfants de la lower middle class qui espèrent ainsi contrecarrer la descente sociale qui les menace. Or le développement rapide de la Chine et de l'Inde se traduit par la constitution d'une vaste classe moyenne éduquée qui a les moyens d'émigrer si elle devait être bloquée dans son ascension dans son propre pays.

\section{Les émigrés comme ressource. Politiques des pays du Sud vis-à-vis de leurs émigrés}

10 Depuis des décennies, le développement et l'emploi sont les leitmotiv des agendas économiques et sociaux des pays arabes et de la Turquie. Ils reconnaissent généralement que le départ à l'étranger d'une main-d'œuvre en surplus ne peut qu'alléger la pression sur le marché du travail et indirectement sur l'État. Leur position vis-à-vis de l'émigration varie de l'incitation au laisser-faire. Elle varie également avec le niveau de qualification des émigrants, les gouvernements étant plutôt enclins à encourager une émigration peu qualifiée et à s'alarmer de l'émigration des diplômés, mais devant l'importance du chômage des diplômés en Égypte et au Maroc, un mouvement se dessine en sens opposé.

11 À partir des années 1960 (Turquie, Maroc, Tunisie, Algérie) et 1970 (Égypte, Jordanie), les gouvernements de plusieurs pays ont activement facilité l'émigration de leurs ressortissants, et certains en ont fait une partie intégrante des stratégies de croissance 
énoncées dans leurs plans de développement. Le Maroc, dès le plan quinquennal de 1968, fixait comme objectif le plus grand nombre possible d'émigrés afin de ne garder au pays que le plus petit nombre possible de chômeurs, et d'attirer avec l'épargne des émigrés des ressources financières pour l'économie nationale. Le Maroc n'a jamais abandonné cette ligne, même lorsqu'à partir de 1973 les gouvernements européens ont l'un après l'autre fermé leurs portes à l'immigration de travailleurs. En affirmant en même temps sa réticence à l'intégration des émigrés marocains en Europe et son refus de reconnaître la double nationalité, le roi Hassan II était parfaitement cohérent: l'émigration est une exportation, et s'il faut la promouvoir, ce doit être pour le bénéfice du Maroc. Dans son discours du Trône de 2005, le roi Mohammed VI est allé plus loin que son père en annonçant l'octroi de la nationalité marocaine aux descendants directs d'émigrés.

D'autres pays ont une politique plus réservée vis-à-vis des flux d'émigration. L'Algérie, qui suspendit unilatéralement son émigration en 1973, est restée longtemps partagée entre deux positions : fort de sa richesse pétrolière et gazière par rapport à laquelle les remises d'épargne des émigrés sont quantité négligeable, et soucieux de rester à l'avant-garde de l'« anti-impérialisme ", son gouvernement a plus d'une fois dénoncé l'émigration comme une forme de dépendance post-coloniale. Mais en même temps, incapable d'assurer le plein-emploi sur un marché du travail miné par un taux de chômage oscillant entre 20 et 30 \% depuis l'Indépendance jusqu'à présent, il s'est bien gardé d'organiser le retour de ses émigrés. Cette ambiguïté perdure: alors qu'une ordonnance de 2005 modifiant le code de la nationalité dispose que tout enfant né de mère algérienne jouit de la nationalité algérienne, à la naissance, au même titre qu'un enfant né de père algérien, le Président Bouteflika remettait en cause le principe de la double nationalité dans un discours de 2006. En des termes différents, le Liban affiche une certaine inquiétude devant l'hémorragie que représente un flux d'émigration que la fin de la guerre civile (1975-1989) n'avait pas tari, et que la guerre de l'été 2006 risque fort d'avoir amplifié. Entre 1987 et 1996, ce sont près d'un cinquième des adultes ( $22 \%$ des hommes et $15 \%$ des femmes entre 25 et 40 ans) qui ont émigré, et dans les cinq années suivantes, on enregistrait encore 1 départ pour 10 adultes $^{8}$.

Les pays arabes (sauf la Libye qui n'a pas de communauté émigrée significative) et la Turquie ont tous établi des institutions, souvent des ministères, pour encadrer la relation du pays avec ses expatriés. Ces institutions suivent deux lignes, l'une économique et l'autre culturelle. La ligne économique consiste à maximiser le bénéfice que le pays peut tirer de l'argent de la diaspora. Il s'agit d'inciter les émigrés à transférer le maximum d'épargne vers leur pays d'origine et à y réaliser le plus possible d'investissements. La ligne culturelle répond au constat que les émigrés, dans leur grande majorité, ne retournent pas au pays sauf pour de courts séjours. Dans les pays occidentaux où le regroupement familial a été reconnu comme un droit fondamental, ils ont amené leurs épouses et ont procréé des enfants. L'arrivée des secondes générations s'est accompagnée d'une transition culturelle au sein de la famille, les parents ayant été élevés dans le pays d'origine et des enfants dans le pays d'accueil. Dans le but de maintenir le lien de la seconde génération avec le pays d'origine des parents, les ministères et les institutions publiques chargées de l'émigration ont donc développé une série d'activités permettant à cette génération de raviver son identité arabe ou turque (cours de langue, vacances au pays) et musulmane (encadrement de la religion). En particulier, ils voient dans la formation des imams une garantie contre 
l'auto-désignation, au sein des populations émigrées, d'éléments susceptibles de troubler l'ordre public et d'offrir une proie facile aux mouvements extrémistes ${ }^{9}$.

\section{La hausse du niveau d'éducation et la baisse du coût de transport contribuent à la mondialisation des migrations}

Outre les possibilités d'emploi, deux autres facteurs encouragent la migration, la baisse du prix des transports et la hausse du niveau d'éducation. Dans les deux cas, la situation a profondément changé depuis le début de la crise en 1974. Le coût de la traversée de la Méditerranée ne représente plus que quelques journées de salaire. Il encourage à tenter l'aventure, sachant que le retour ne sera pas aussi onéreux qu'auparavant.

D'autre part, le niveau de formation des jeunes s'est considérablement amélioré dans les pays du Sud. Ceci est allé de pair avec une mutation démographique puisque la fécondité est maintenant inférieure à 2,5 enfants dans l'ensemble du Maghreb et à 3,5 en Égypte. Comme les personnes âgées sont peu nombreuses, les jeunes adultes issus des familles nombreuses des années 1950 à 1980 peuvent facilement s'occuper de leurs parents et comme ils se marient tard et font de petites familles, ils ne sont plus dans la nécessité de trouver à tout prix des moyens de subsistance. S'ils migrent, ce n'est plus pour faire survivre leur famille, désormais de taille réduite, mais pour se réaliser car l'éducation et les médias ont élargi leur champ d'attentes. Les migrations dictées par l'impératif de la subsistance proviennent de plus loin, du Sahel et de la côte de Guinée, mais elles viennent le plus souvent échouer en Afrique du Nord d'où elles parviennent difficilement à franchir la Méditerranée.

\section{Les PSEM sont désormais eux aussi confrontés à l'immigration et à l'intégration}

\section{Les Pays du sud et de l'est de la Méditerranée (PSEM), pays d'immigration ${ }^{10}$}

L'une des conséquences inattendues de cette transformation est l'apparition d'immigrants dans les pays du PSEM qui sont aujourd'hui tous devenus des pays d'immigration. Certains d'entre eux comptent plusieurs centaines de milliers d'immigrés réguliers : la Libye, qui reste un pays d'emploi pour de nombreux Égyptiens, Soudanais, Tunisiens, Tchadiens... quoiqu'à une moindre échelle qu'avant les années d'embargo international (1992-1999) ; la Jordanie, avec plus de 225000 Égyptiens, pour la plupart ouvriers agricoles, et quelques dizaines de milliers d'Asiatiques qui travaillent notamment dans les zones franches industrielles; le Liban, où plus de 50000 permis de résidence sont délivrés chaque année à des femmes immigrées du Sri Lanka et des Philippines, employées dans les services domestiques. S'y ajoutent d'autres migrations de grande ampleur se comptant chacune en centaines de milliers, bien qu'elles ne laissent aucune trace dans les statistiques: les Soudanais en Égypte, les Irakiens en Jordanie et les travailleurs temporaires et irréguliers syriens au Liban (avant l'évacuation du Liban par l'armée syrienne au printemps 2005, en tout cas). 

montée de l'immigration irrégulière dans les pays arabes riverains de la Méditerranée qui restent de grands foyers d'émigration, ainsi qu'en Turquie. Il s'agit le plus souvent d'une immigration qualifiée de transit parce qu'elle se compose de personnes qui se destinaient initialement à des pays plus lointains, l'Europe ou l'Amérique du Nord, à la porte desquels elles se sont trouvées bloquées, faute de remplir les conditions d'obtention d'un visa. Cette immigration est généralement perçue comme indésirable par les gouvernements des pays de transit, car elle met au défi des économies déjà minées par le sous-emploi des nationaux, et des appareils administratifs mieux adaptés à traiter de l'émigration de nationaux que de l'immigration d'étrangers.

L'ensemble du Maghreb est désormais devenu une région de transit. Cela a commencé par le Maroc, le plus proche de l'Europe, puis s'est étendu à des pays de plus en plus lointains au fur et à mesure que les contrôles se renforçaient sur les routes les plus courtes. Successivement l'Algérie, la Tunisie, la Libye et maintenant la Mauritanie, sont devenus lieux d'embarquement pour des migrants venus du Sud du Sahara; le Liban, la Turquie et la Syrie pour ceux venus du Moyen-Orient ou d'Asie centrale. Comme il faut parfois très longtemps pour embarquer, et que tous n'y parviendront pas, les migrants qui étaient initialement en transit deviennent des immigrés, le plus souvent irréguliers, et entrent sur le marché local de l'emploi. Ils y forment une main-d'œuvre particulièrement vulnérable, et donc flexible, dont l'économie locale tire souvent profit. Les effectifs concernés se chiffrent en dizaines de milliers dans chacun des pays du Maghreb et en Turquie. Mais le phénomène ne se limite pas à ces pays. Il s'étend à l'Égypte, pays de transit pour de nombreux migrants soudanais, somaliens et éthiopiens. Le Yémen lui-même, l'un des pays les plus

pauvres du monde mais situé à la porte de pays qui sont parmi les plus riches, est devenu un lieu de transit à grande échelle pour des migrants originaires de la Corne de l'Afrique qui cherchent à gagner le Golfe. En raison du renforcement des pressions migratoires en Afrique, d'un côté, et du durcissement des conditions d'accès à l'Europe ou à l'Amérique du Nord, de l'autre, il y a tout lieu de penser que ce type d'immigration ne peut que prendre de l'ampleur dans les années qui viennent.

\section{Protectionnisme et absence de projet d'intégration des immigrés au Sud et à l'Est}

Tous les pays arabes ont aujourd'hui une politique consistant à réserver l'emploi à leurs nationaux. C'est une situation nouvelle. À la suite de la Guerre du Golfe de 1990-91, les pays du Golfe, les premiers, ont développé des politiques d'indigénisation du travail. Elles sont doublement protectionnistes. D'une part, en accroissant les barrières à l'entrée (octroi de plus en plus difficile des visas) et au séjour (limitation ou suppression pure et simple du regroupement familial, non-éligibilité des étrangers à la sécurité sociale et à l'éducation gratuite), elles visent à infléchir l'offre de travail étranger. D'autre part, en réservant par la loi toute une liste d'emplois aux nationaux et en taxant les entreprises à l'embauche d'étrangers, elles agissent sur la demande ${ }^{11}$. Cependant, la désaffection de la main-d'œuvre nationale pour les emplois salariés du secteur privé et celle des employeurs pour cette même main-d'œuvre (qui n'accepte pas les basses rémunérations offertes aux immigrés) se sont jusqu'à présent conjuguées pour mettre en échec ces politiques d'indigénisation. 
21 Nous l'avons vu, tous ces pays sont confrontés à la montée des migrations irrégulières. Deux d'entre eux seulement, le Maroc en 2003 et la Tunisie en 2004, ont construit des instruments juridiques pour y répondre, sous forme de lois sur l'entrée, la résidence et la sortie des étrangers. Ces lois prévoient des peines sévères applicables aux trafiquants mais aussi aux migrants eux-mêmes, tout en ne contenant aucune disposition qui protégerait ceux-ci contre un mauvais traitement de la part des employeurs ou de l'administration. Elles ont soulevé la critique des organisations de défense des droits de l'homme, qui y ont vu à la fois un cadre susceptible d'aggraver un drame humanitaire déjà considérable, et un acte d'abdication devant les pressions de l'Europe, au détriment des bonnes relations avec les voisins africains. C'est dans ce contexte que les gouvernements des pays du Maghreb préconisent une approche globale des migrations: la prévention de la migration irrégulière et la réouverture de canaux légaux de migration économique doivent être deux facettes inséparables de toute politique cohérente de migration en Méditerranée ${ }^{12}$.

Quand des migrants temporaires ou en transit s'installent, la question de leur intégration dans le pays d'accueil se pose. La Commission européenne a ainsi recommandé que les immigrés acquièrent progressivement des droits et des devoirs qui leur assurent à l'issue d'un certain nombre d'années un traitement équivalent à celui des nationaux du pays hôte (droit au regroupement familial, accès au travail et égal traitement sur le lieu du travail, citoyenneté civique, lutte contre les discriminations, notamment raciale et dans l'emploi). Aucun pays arabe n'a de projet de ce type. Intégrer les immigrés dans la société, qu'ils soient arabes on non arabes, n'est nulle part à l'ordre du jour. La Turquie, de ce point de vue, se distingue par une volonté d'intégration des immigrés turcs ethniques ${ }^{13}$.

\section{L'augmentation des mobilités transméditerranéennes}

La fermeture des frontières dans la plupart des pays européens à partir de la crise de 1974 a transformé la perception de l'immigration. Jusqu'alors, ceux qu'on appelait des travailleurs migrants étaient censés venir travailler au Nord pour quelques années puis revenir dans leur pays. Ce comportement était qualifié de noria en France et de Gastarbeiter en Allemagne. Après 1974, les migrants sont devenus des immigrés restant sur place et obtenant le bénéfice du regroupement familial. Le mythe d'une immigration «zéro » a renforcé cette nouvelle perception des migrations. Dès lors, le problème de l'immigration devenait celui de l'intégration. Or la circulation migratoire a continué, contournant les obstacles légaux, la migration de court terme devenant même une forme de mobilité de plus en plus fréquente. Des migrants ont continué à arriver et d'autres à repartir. Depuis quelques années, la rotation s'est accélérée, dopée par la baisse des coûts de déplacement en regard des salaires. En Allemagne, par exemple, on a enregistré en 2002658000 entrées et 505000 sorties, parmi lesquelles 58000 entrées de Turcs et 37000 sorties $^{14}$. En France, sur 100 personnes officiellement entrées entre 1990 et 1994, seules 55 étaient encore présentes au recensement de 199915. Toujours en France, un comptage sur les années 1994 à 1997 a montré que seuls $42 \%$ des étudiants renouvelaient leur titre donc demeuraient plus d'une année ${ }^{16}$.

Cette mobilité accrue ou retrouvée va de pair avec des mouvements plus importants et plus difficiles à mesurer. La France a enregistré en 2003 plus de 80 millions de séjours d'étrangers pour au moins une nuit et a émis plus de deux millions de visas de trois 
mois, accordés pour motif de tourisme ou d'affaires, mais qui peuvent être détournés en travail non-déclaré. Plus difficile à mesurer d'une part parce qu'on mesure très mal les immigrés qui repartent, d'autre part parce que dans la zone grise de la clandestinité, les séjours temporaires de travailleurs sans permis se sont accrus dans des proportions importantes, en particulier à partir de l'Ukraine, de la Moldavie et des Balkans. Ces mouvements ne sont pas seulement dirigés vers l'UE. Grâce au faible coût et à la facilité de la traversée de la mer Noire, de nombreux Ukrainiens, Russes et Géorgiens munis d'un visa de tourisme viennent travailler à titre temporaire en Turquie où la demande d'emploi de techniciens du bâtiment ou du tourisme est importante et les salaires supérieurs à ceux du pays d'origine.

La mobilité concerne aussi les immigrés installés depuis longtemps dans le pays d'accueil car ils peuvent plus facilement retourner au pays d'origine par exemple à l'occasion des congés. Plus généralement, la migration crée plus fortement qu'auparavant des liens entre un pays d'arrivée et des pays ou régions de départ, d'autant que les contacts entre pays de départ et d'arrivée sont facilités par les moyens modernes de communication (paraboles, internet, portables). Les liens se resserrent donc entre les deux rives de la Méditerranée. Ils mènent à l'émergence de ce que l'on peut qualifier de bi-nationalité où les individus participent à deux cultures et les maitrisent, ce qui transforme la question de l'intégration. Cette bi-nationalité est aussi le fait de l'ancienneté de la migration qui a accru le nombre des descendants de seconde et troisième génération. Elle se manifeste enfin par des doubles nationalités qu'encouragent les lois récentes passées dans les pays du Maghreb comme on l'a vu avec l'exception de l'Algérie, sans doute contournée par les intéressés.

\section{NOTES}

1. En ne tenant pas compte des migrations entre États de l'UE (données de l'OCDE pour l'année 2004).

2. La Pologne et les trois États baltes (EUROSTAT, Chronos Database).

3. Leonid Rybakovsky and Sergey Ryazantsev, International migration in the Russian Federation (UN/POP/MIG/2005/11).

4. À l'exception d'Israël et de la Libye qui ont tous les deux un solde migratoire positif.

5. H. Zlotnik, "International migration 1965-96: an overview", Population and Development Review, 1998, 24, p. 429-468.

6. Notamment des musulmans chinois dont la minorité est forte de 60 millions de personnes.

7. Sauf quand on les recrute selon ce critère, ce qui a été le cas pour l'émigration algérienne des années 1960 et 1970.

8. $9 \%$ en 1997-2002. Robert Kasparian et Choghig Kasparian, «Émigration, emploi et conditions économiques. Le cas du Liban ", communication présentée au séminaire Forecasting Economic International Migration in the Euro-Mediterranean Area, 2005-2025, Istanbul, 2006 (à paraître sur www.carim.org). 
9. Ph. Fargues (dir.), 2005b, conclusions du séminaire «Politiques d'intégration : la vision des pays tiers méditerranéens ", Tunis, 12-15 décembre 2005, http://www.carim.org/Publications/ 200512-CARIM-TunisSem.pdf.

10. «PSEM » : Pays du Sud et de l'Est de la Méditerranée.

11. Shah Nasra 2006, « Restrictive Labour Immigration Policies in the Oil-Rich Gulf : Implications for Sending Asian Countries » United Nations Expert Group Meeting on International Migration and Development in the Arab Region, Beyrouth, 15-17 mai 2006, http://www.un.org/esa/population/ publications/EGM_Ittmig_Arab/Itt mig_Arab.htm

12. J.-P. Cassarino 2005, «Website guide on Europe's Migration Policy in the Mediterranean », CARIM Analytical and Synthetic Notes, 2006/10, http://www.carim.org/publications/CARIM-AS05_10-Cassarino.pdf

13. K. Kirisci, 2004, « Asylum, Immigration, Irregular migration and Internally Displacement in Turkey : Institutions and Policies ", CARIM Analytical and Synthetic

Note 2004/3, http://www.carim.org/publications/CARIM-AS04_03-Kirisci.pdf

14. J. Lang et H. Le Bras, Immigration positive, Paris, éd. Odile Jacob, 2006, p. 229-230.

15. Tableaux thématiques du recensement de 1999 : population immigrée, population étrangère, Paris, INSEE, 2001.

16. $X$. Thierry : «La fréquence du renouvellement des premiers titres de séjour », Population, 56, $\mathrm{n}^{\circ}$ 3, 2001, p. 451-468.

\section{RÉSUMÉS}

Des « quatre libertés » que l'UE entend développer avec son voisinage, les migrations sont la plus mal assurée et assumée. Le sommet de Barcelone+10 de novembre 2005 a pourtant rappelé la nécessité de «créer un espace de coopération mutuelle en matière de migrations ». De fait, l'UE est désormais la première région d'immigration du monde, avec une migration nette de $+2,8$ millions en 2004 (contre 1,2 million pour l'Amérique du Nord - même si l'Amérique du Nord compte 24 millions de personnes qui vivent dans un pays où elles ne sont pas nées contre 18 millions pour l'Union européenne). Le trait va se renforcer avec les besoins de main-d'œuvre d'une Europe de plus en plus âgée. En dépit de la globalisation croissante des routes de la migration, l'attraction de l'UE s'exerce d'abord sur sa périphérie, ce qui, joint à la jeunesse des pays Sud-méditerranéens, définit les conditions d'une complémentarité stratégique. Mais cette complémentarité est entravée par des politiques nationales qui raisonnent encore en termes de migrations (définitives), là où les réalités sociales conduisent à l'idée de mobilités. Dans cet angle de vue, l'article esquisse des outils innovants comme les « migrations qualifiantes ", associant la mobilité à la formation de la main-d'œuvre venue du Sud et dont l'Europe a besoin.

Among the four freedoms that the European Union tries to enhance in its relations with its neighbourhood, the freedom of movement (international migrations) is the most badly treated and accepted. Yet, the Barcelona +10 Summit, which took place in Novembre 2005, put the stress on the necessity to open a space of mutual cooperation in the field of international migrations. Actually, the Euroopean Union has become the most attractive region in the world for the migrants, with a net inward flow of 2.8 million migrants in 2004 (1.2 in the United States). This tendency is most likely to strengthen with the rapid ageing of the European labour force. In spite of the growing globalisation of the migration routes, the European Union exerts mainly its 
attraction on its geographical peripheries. Taking account of the high number of young people in the Mediterranean countries, such a situation is based on an obvious North South strategic complementarity. Nevertheless, this complementarity is hampered by national policies mainly based on the idea that the migrants try to leave their country of origine for ever. Such a representation is not correct : more and more migrants are involved in a process of permanent mobility between the north and the south bank of the Mediterranean. Taking account of this tendency, the authors underline the idea that the international migration could increase the professionnal skills of the migrants. From this point of view, they propose to make the needs of the EU and of the countires of origin meet by associating the mobility of the migrants and their training in order to enhance their level of education.

INDEX

Mots-clés : Union européenne, flux migratoires, démographie, emploi, éducation, mobilité Index géographique : Méditerranée, Afrique du Nord

\section{AUTEURS}

PHILIPPE FARGUES

CARIM, Institut universitaire européen de Florence

HERVÉ LE BRAS

EHESS, INED 